\title{
PROBLEMS FOR SOLUTION
}

$P$ 110. Find the order, class, number of nodes, and number of cusps of the curve

$$
x_{1}^{2 / 3}+x_{2}^{2 / 3}+x_{3}^{2 / 3}=0
$$

in the complex projective plane.

\section{H.S. M. Coxeter, University of Toronto}

$P$ 111. Let $G$ denote the set of polynomials $f$ of the form $f(t)=t^{n}+\sum_{k=1}^{n} c_{k} t^{n-k}$ which have $n$ real roots in $[-1,1]$, not necessarily distinct. Let $g_{k}(k=0, \ldots, n)$ denote the following element of $G: g_{k}(t)=(t+1)^{k}(t-1)^{n-k}$. Prove that $G$ is a subset of the convex hull of the $g_{k}$.

Chandler Davis, University of Toronto

$P$ 112. It is known from results of $\mathrm{L}$. Moser and $\mathrm{J}$ J. Lambek [Proc. Amer. Math. Soc. 4 (1953), 544-545], among others, that every monotone, real-valued, multiplicative arithmetic function $f \neq 0$ is of the form $f(n)=n^{\alpha}$ for some non-negative real number $\alpha$. Prove the stronger result that every monotone, real-valued, generalized multiplicative [Amer. Math. Monthly 72 (1965), 1140] arithmetic function $f \neq 0$ is of the form $f(n)=n^{\alpha}$ for some non-negative real number $\alpha$.

Albert A. Mullin, University of California 